\title{
Early maternal Zika infection predicts severe neonatal neurological damage: results from the prospective Natural History of ZIKA Virus Infection in Gestation (NATZIG) cohort study
}

\author{
Conrado Coutinho ${ }^{1}$, Silvia Fabiana Negrini $^{1}$, Daniel Araujo ${ }^{2}$, Sara Teixeira ${ }^{1}$, Fabiana \\ Amaral $^{1}$, Marília Carolina Moro ${ }^{1}$, Juliana Fernandes ${ }^{1}$, Márcia Motta ${ }^{1}$, Bento Negrini ${ }^{1}$, \\ Carla Andréa Caldas ${ }^{1}$, Adriana Anastasio ${ }^{1}$, João Marcello Furtado ${ }^{1}$, Adriana Aparecida \\ Bárbaro $^{1}$, Aparecida Yamamoto ${ }^{1}$, Geraldo Duarte ${ }^{1}$, and Marisa Márcia Mussi-Pinhata ${ }^{1}$ \\ ${ }^{1}$ Universidade de Sao Paulo Hospital das Clinicas da Faculdade de Medicina de Ribeirao \\ Preto \\ ${ }^{2}$ Secretary of Health, Ribeirão Preto
}

June 1, 2020

\begin{abstract}
Objective: To define the prevalence of adverse outcomes according to the gestational age of maternal infection in a large cohort of ZIKV-infected Brazilian women and their infants. Design: Prospective, population-based cohort study. Setting: Ribeirão Preto's region private and public health facilities. Population: All pregnant women with confirmed ZIKV-infection and their infants. Methods: Prenatal/early neonatal data were obtained for all pairs. A subgroup of infants had cranial ultrasonography, eye fundoscopy, hearing, neurological exam, and Bayley III screening test within 3 months of age. Main Outcome Measures: Prevalence of pregnancy losses, anomalies detected at birth or within 3 months according to the gestational age of infection. Results: 511 women with ZIKV were identified from a total of 1116 women with flavivirus-type symptoms. Pregnancy losses 24/511(4.7\%) and/or ZIKV-related anomalies occurred in 43/511(8.4\%) mothers. Microcephaly or other CNS malformations were diagnosed in $1 / 4(25.0 \%)$ of the stillbirths and 19/489[3.9\%;CI95\%:2.5-5.9] of the live-born infants, with 13/513[2.5\%;CI95\%:1.5-4.3] neonates presenting major signs of CZS. Fetal abnormalities were 14.0(CI95\%:7.6-26.0) times more likely with gestational infection $<11$ weeks. Among 280 asymptomatic evaluated infants, 2/155(1.3\%) had eye abnormalities, 1/207(0.5\%) CNS imaging significant findings, and 16/199(8\%) neurological alert signs. Conclusions: This prospective, population-based study represents the largest Brazilian cohort of ZIKV in pregnancy. Congenital anomalies potentially associated with CZS are less frequent than previously thought. There is a strong relationship between the gestational age of infection $(<11$ weeks $)$ and worse early infant prognosis. A notable proportion of apparently asymptomatic newborns can present with subclinical findings within 3 months of age. Funding: FAEPA/SES-SP
\end{abstract}

Manuscript title: Early maternal Zika infection predicts severe neonatal neurological damage: results from the prospective Natural History of ZIKA Virus Infection in Gestation (NATZIG) cohort study.

\section{Authors and highest degrees :}

Conrado Milani Coutinho, MD, DsC ${ }^{1}$

Silvia Fabiana Biason de Moura Negrini, $\mathrm{DsC}^{2}$

Daniel Cardoso de Almeida e Araujo, MD, $\mathrm{DsC}^{3}$

Sara Reis Teixeira, MD, $\mathrm{DsC}^{4}$ 
Fabiana Rezende Amaral, $\mathrm{MD}, \mathrm{MsC}^{2}$

Marília Carolina Razera Moro, $\mathrm{MD}^{1}$

Juliana Dias Crivelenti Pereira Fernandes MD²

Márcia Soares Freitas da Motta, MD, $\mathrm{MsC}^{2}$

Bento Vidal de Moura Negrini, MD, $\mathrm{DsC}^{2}$

Carla Andréa Cardoso Tanuri Caldas, $\mathrm{MD}^{5}$

Adriana Ribeiro Tavares Anastasio, $\mathrm{MsC}, \mathrm{DsC}^{6}$

João Marcello Furtado, MD, $\mathrm{DsC}^{7}$

Adriana Aparecida Tiraboschi Bárbaro, $\mathrm{MD}, \mathrm{MsC}^{2}$

Aparecida Yulie Yamamoto, MD, $\mathrm{DsC}^{2}$

Geraldo Duarte, MD, DsC ${ }^{1}$

Marisa Márcia Mussi-Pinhata, MD, $\mathrm{DsC}^{2}$

for the NATZIG cohort study team.

\section{Affiliation:}

1. Department of Obstetrics and Gynecology, Ribeirão Preto Medical School University of São Paulo, Ribeirão Preto, SP, Brazil.

2. Department of Pediatrics, Ribeirão Preto Medical School University of São Paulo, Ribeirão Preto, SP, Brazil.

3. Epidemiology and Disease Control Division, Department of Public Health and Surveillance, Secretary of Health, Ribeirão Preto, SP, Brazil.

4. Department of Imaging, Hematology and Oncology, Ribeirão Preto Medical School University of São Paulo, Ribeirão Preto, SP, Brazil.

5. Rehabilitation Center of Clinics Hospital at the Ribeirão Preto School of Medicine, Ribeirão Preto, SP, Brazil.

6. Department of Health Sciences, Ribeirão Preto Medical School, University of São Paulo, Brazil.

7. Division of Ophthalmology, Ribeirão Preto Medical School, University of São Paulo, Ribeirão Preto, SP, Brazil.

Corresponding author: Conrado Milani Coutinho

Hospital das Clínicas da Faculdade de Medicina de Ribeirão Preto da Universidade de São Paulo. Campus Universitário s/no ${ }^{\circ}$ CEP: 14048-900, Ribeirão Preto, SP, Brazil. Phone: +55 16 3602-2925. Fax: +55 16 3602-1549. Email: cmcoutinho@hotmail.com

Running title: Early maternal Zika infection and severe outcomes.

Word count of main text: 3435 words.

\section{Abstract :}

Objective: To define the prevalence of adverse outcomes according to the gestational age of maternal infection in a large cohort of ZIKV-infected Brazilian women and their infants.

Design: Prospective, population-based cohort study. 
Setting: Ribeirão Preto's region private and public health facilities.

Population: All pregnant women with confirmed ZIKV-infection and their infants.

Methods : Prenatal/early neonatal data were obtained for all pairs. A subgroup of infants had cranial ultrasonography, eye fundoscopy, hearing, neurological exam, and Bayley III screening test within 3 months of age.

Main Outcome Measures: Prevalence of pregnancy losses, anomalies detected at birth or within 3 months according to the gestational age of infection.

Results : 511 women with ZIKV were identified from a total of 1116 women with flavivirus-type symptoms. Pregnancy losses 24/511(4.7\%) and/or ZIKV-related anomalies occurred in 43/511(8.4\%) mothers. Microcephaly or other CNS malformations were diagnosed in $1 / 4(25.0 \%)$ of the stillbirths and 19/489[3.9\%;CI95\%:2.5-5.9] of the live-born infants, with 13/513[2.5\%;CI95\%:1.5-4.3] neonates presenting major signs of CZS. Fetal abnormalities were 14.0(CI95\%:7.6-26.0) times more likely with gestational infection $<11$ weeks. Among 280 asymptomatic evaluated infants, 2/155(1.3\%) had eye abnormalities, 1/207(0.5\%) CNS imaging significant findings, and 16/199(8\%) neurological alert signs.

Conclusions: This prospective, population-based study represents the largest Brazilian cohort of ZIKV in pregnancy. Congenital anomalies potentially associated with CZS are less frequent than previously thought. There is a strong relationship between the gestational age of infection $(<11$ weeks $)$ and worse early infant prognosis. A notable proportion of apparently asymptomatic newborns can present with subclinical findings within 3 months of age.

Funding: FAEPA/SES-SP

Keywords: ZIKA virus, pregnancy, infant, adverse outcomes, congenital Zika syndrome.

Twitable abstract:

ZIKV and pregnancy: adverse outcomes are less common, more prevalent for 1st trimester infections and potentially subclinical.

\section{INTRODUCTION}

Zika virus (ZIKV) infection is particularly important when acquired during pregnancy. Perinatal adverse outcomes have been linked to pregnancy losses or fetal neurological damage ${ }^{1,2}$. Adverse effects following maternal ZIKV infection have been previously reported, but there is limited data available from large cohorts of ZIKV-infected mother-infant pairs. A wide variety of inclusion criteria and definitions of adverse outcomes have been used for prevalence estimates of abnormalities ranging widely from $5-46 \%^{3-13}$. Further, the impact of the gestational age of maternal infection on the risk of fetal abnormalities has not yet been fully defined.

Although the epidemic phase of ZIKV infection is over, there is still interest on enhancing knowledge about mother-to-child transmission and its consequences to the infant, since the endemic spread is continuing globally ${ }^{14}$ and could reemerge as a public health problem due to socioeconomic or climatic changes ${ }^{15}$.

In 2016, a large cohort of ZIKV-infected symptomatic pregnant women and their infants was composed in Ribeirão Preto metropolitan region, Brazil. Our primary objective was to investigate the prevalence of adverse pregnancy and early neonatal outcomes with an emphasis on pregnancy losses, microcephaly, and Congenital Zika Syndrome (CZS) as functions of the gestational age of maternal infection. A subset of infants had received an additional specialized evaluation within three months of life, allowing us to estimate the probability of occurrence of early-presenting occult/mild end-organ disease among infants who had not been identified affected at birth.

\section{METHODS}

The Natural History of ZIKA Virus Infection in Gestation (NATZIG) project is a prospective populationbased cohort study undertaken in the entire Ribeirão Preto region (population 1,720,469) ${ }^{16}$, in northeastern 
São Paulo state, Brazil. In this paper, we enrolled symptomatic ZIKV-infected pregnant women and -exposed infants during the 2015-2016 ZIKV outbreak. All study procedures received ethical approval (Processes \#7404/2016, \#5914/2017), and written informed consent was obtained from all participants.

\section{Study Population}

Every pregnant woman who contacted a health facility with Flavivirus-like symptoms and were reported to the local Disease Control Division (DCD) and had blood (within 5 days of symptoms) and urine (within 8 days) taken for RNA-ZIKV ${ }^{17}$ testing by Adolfo Lutz Institute. ZIKV infection was confirmed in women if there was detectable ZIKV-RNA in blood, urine, amniotic fluid, or feto-placental tissue after pregnancy loss.

Most patients received prenatal care and delivered in various institutions without knowing their ZIKVRNA status, as the sudden pandemic meant that demands on the single reference laboratory was very high, leading to delay in results (median 39 days). A taskforce (NATZIG study team, Ribeirão Preto DCD, and the São Paulo State Secretary of Health) contacted all mothers and obtain pregnancy, delivery, and neonatal information from medical records, their family physicians and directly from patients.

Procedures and Definitions

Maternal demographic, clinical, laboratory, obstetrical, and neonatal data were recorded in standardized forms. Prenatal routine maternal testing was done for toxoplasmosis, hepatitis B and C, human immunodeficiency virus (HIV), and syphilis. Gestational age was determined in completed weeks by obstetrical estimation $^{18}$. Spontaneous abortions and stillbirths were defined as embryo or fetal losses that occurred before and after 20 completed weeks of pregnancy, respectively.

Newborn data were extracted from medical records. Birth weight and head circumference measured before hospital discharge were classified according to the INTERGROWTH-2 $1^{\text {st }}$ criterion ${ }^{19}$ as recommended by the PAHO. Microcephaly was defined as a head circumference $(\mathrm{HC})>2 \mathrm{z}$ scores below the appropriate mean for a given age, sex, and gestational age, and severe microcephaly was defined as an $\mathrm{HC}>3 \mathrm{z}$ scores below these standards ${ }^{19,20}$. All infants were initially classified according to the presence of microcephaly, neonatal neurologic dysfunctions, and other birth defects coded as major or minor ${ }^{21}$.

Combined neonatal abnormalities detected in fetuses/infants were defined as potentially related to congenital ZIKV infection both according to Honein et al. ${ }^{6}$ and Pomar et al. ${ }^{1}$ criteria as mild/moderate or severe complications compatible with CZS. Typical CZS phenotype was defined as those classified by the former authors as "brain abnormalities with or without microcephaly" and by the latter as those presenting "severe complications compatible with CZS or fetal loss". We did not to exclude the first-trimester spontaneous abortions from the adverse outcomes but excluded the fetuses/newborns with other known causes for the CNS anomalies from the typical CZS phenotype definition. Both decisions were taken to allow a better understanding of the complete investigation of cases.

Additional assessments were planned for all infants with microcephaly and other major neurologic findings and those who were born or referred within 3 months of life as follows: Hammersmith Neonatal Neurological Examination (HNNE) ${ }^{22}$; pediatric evaluations at 3-6 weeks and $3+1$ months focusing on clinical and neurological findings and the developmental milestones; Bayley-III scale of infant and toddler development screening test ${ }^{23}$ performed by a certified examiner; hearing screening using both Transient Otoacoustic Emissions (TOAE) and automated auditory brainstem evoked responses (AABR-35dBHL); cranial ultrasonography and/or other neuroimaging studies performed by an experienced physician and reviewed by two board-certified radiologists; complete eye examination including indirect ophthalmoscopy by ophthalmologist.

The HNNE optimality compound score is calculated for each of six categories (tone, tone patterns, reflexes, movements, deviant signs, behavior) of a neurological evaluation containing 34 items. Scores between 30.5 and 34 are found in more than $95 \%$ of low risk term infants ${ }^{22}$. For the purpose of this analysis, an optimality score $<30.5$ at birth were considered suboptimal. For infants with no HNNE complete evaluation, a short neurological exam proforma of this test with 13 categories was applied ${ }^{24}$ and the presence of any abnormal 
sign was considered suboptimal. Regarding the Bayley screening test, infants were tested according to the application guidelines in five domains: cognitive, communication (receptive and expressive), and motor (fine and gross). Scores were classified as competent or emerging/at risk in each domain ${ }^{23}$. An infant with at least two of the neurologic/neurodevelopmental evaluations categorized as suboptimal, abnormal, emerging or at risk was classified with a possibleneurologic abnormality.

Laboratory Testing

Infants with findings suggestive of congenital infections were submitted to testing for syphilis (treponemal and reaginic tests) and toxoplasmosis (IgM and IgG ELISA). Also, blood, saliva, urine and/or liquor were tested for enterovirus, parvovirus, HHV-6, HSV-I, HSV-II, and CMV via PCR assays. Infants with CNS abnormalities for whom a blood sample had been obtained within three months of age were tested for antiIgM ZIKV antibodies (ZIKV Detect ${ }^{T M} 2.0$ IgM Capture Elisa, InBios International, Inc. Seattle, WA, USA), and ZIKV-RNA (CDC Trioplex Real-time RT-PCR Assay).

Data analysis

The general characteristics of subjects were described according to the median, ranges, frequencies, and percentages. A Conditional inference tree, a non-parametric multivariate regression model, using thepartykit library of R 3.5.1 software, was applied to classify groups according to the presence of any adverse outcomes potentially related to ZIKV infection (miscarriages, stillbirths, microcephaly with or without brain abnormities, and brain abnormalities without microcephaly) from gestational age ${ }^{25}$. In general, this method selects, among independent variables, the ones that better classifies the individuals according to the presence of the outcome. In the next step, the algorithm considers all the independent variables to select another (or the same variable previously selected but estimating different cut-off from the first step) that better classifies the individuals according to the outcome and the process extends until there are no more variables selected. The independent variable used by us in these analyses was the gestational age. The same method was used to classify groups more likely to present a typical CZS phenotype as a function of gestational age. Log-binomial regression models were adjusted to estimate relative risks and their respective $95 \%$ confidence intervals. Data were analyzed using SPSS software version 20.0.

Funding

This study was mainly supported by Fundação de Apoio ao Ensino Pesquisa e Assistência do Hospital das Clínicas da Faculdade de Medicina de Ribeirão Preto da Universidade de São Paulo (FAEPA), Brasil, and received partial support from the CVE - Centro de Vigilância Epidemiológica "Prof. Alexandre Vranjac" da Secretaria da Saúde do Estado de São Paulo (SES-SP). The funding sources didn't have any role in the writing, the decision to submit the manuscript for publication or any other aspect pertinent to study conduction. There was no funding from pharmaceutical companies or other agencies. All study data were analysed by the corresponding author, as well as the final responsibility for the decision to submit for publication.

\section{RESULTS}

Overall, 1116 pregnant women were notified between December 2015 and December 2016. Of these, 511 pregnant women had PCR-confirmed ZIKV infection and gave birth in 2016 to 489 newborns. 189/511(37\%) women were referred for prenatal care and delivered at our hospital.

Adverse prenatal and early postnatal infant outcomes

In summary, pregnancy losses and/or birth defects potentially related to ZIKV in newborns occurred in $43 / 511(8.4 \%)$ symptomatic mothers while microcephaly or other CNS malformations were diagnosed in $25.0 \%(1 / 4)$ of the stillbirths and 3.9\%(19/489)[CI95\%:2.5-5.9] of the live-born infants. If only the typical CZS phenotype in infants without co-morbidities was considered, then 12/489(2.9\%;CI95\%:1.7-4.7) infants would be affected by the intrauterine ZIKV-infection or 13/513(2.5\%;CI95\%:1.5-4.3) fetuses. 
Tables S1 and S2 show the overall characteristics and pregnancy outcomes in 511 women. There were 24(4.7\%) pregnancy losses. Postmortem examination of one of the four stillbirths (34 weeks) showed severe CNS malformations. There was one stillbirth of unknown cause. The remaining three fetal losses had no malformations and were likely secondary to premature membrane rupture or placental abruption. Of the remaining 487 women, there were 489 live births.

As shown in Figure 1, of the live-born infants, 33(6.7\%;CI95\%:4.8-9.3) had one or more major abnormalities, and 19(3.9\%;CI95\%:2.5-5.9) had findings potentially related to ZIKV infection (Table 1). Except for three infants (including two that died shortly after birth due to severe CZS anomalies), those with potential ZIKV infection underwent additional postnatal neuroimaging. Malformations of cortical development, brain volume loss, ventriculomegaly, and calcifications were found. All five infants with severe and $16.7 \%(1 / 6)$ of those with moderate microcephaly had eye abnormalities. Two infants without microcephaly had neurological dysfunctions and were diagnosed with brain abnormalities. Of the 17 infants screened for hearing loss, one failed, but we could not confirm this result. Of the 19 infants with findings potentially related to ZIKV infection, 4(21\%) were categorized as mild/moderate and 15(79\%) as severe ${ }^{1}$.

Findings in asymptomatic neonates without microcephaly or evident neurological abnormalities on initial clinical examination

Of the 511 women with ZIKV in the region, there were 470 live-born infants with no microcephaly or other evident neurological findings detected at birth on physical exam. The subsample of $280(59.6 \%)$ infants that were cared for at our hospital, had one or more additional assessments done. Except for maternal age, the 280 infants that were followed up, and their mothers, were not different at baseline from those that were not followed up, suggesting that they are representative of the entire cohort (Table S3). None of 244 tested infants failed the hearing screening. Of the 155 infants submitted to the ophthalmologic exam, two (1.3\%) had ZIKV-related findings: glaucoma or focal epithelium pigment mottling. Of the 207(73.9\%) infants submitted to cranial ultrasonography, one $(0.5 \%)$ had major findings potentially related to ZIKV infection while $47(22.7 \%)$ had minor features. Seventeen of the 199 infants with a neurological evaluation within 3 months of age had a possible abnormality but a normal cranial ultrasonography. One of them had Down syndrome but no underlying comorbidities were detected in the remaining $16(8.0 \%)$ infants. The maternal ZIKV-infection occurred in the first trimester in 11(57.9\%) of the 19 infants with subclinical abnormalities; the rest were in the second trimester. ZIKV-IgM was negative in all 13 infants tested.

Laboratory testing in infants with neurologic abnormalities potentially related to congenital ZIKV infection

A total of 16/19(84.2\%) infants were tested for other congenital infections and were negative for enterovirus (10), parvovirus (12), HSV-I (13), HSV-II (13), and HHV-6 (4). Two dichorionic twins had CMV infection and received antiviral treatment.

Anti-ZIKV IgM was done in 14/17(82.4\%) infants who survived with features potentially related to ZIKV infection. Of these, only four (28.6\%) were positive. One had a previous positive ZIKV-PCR in amniotic fluid, and two had a positive ZIKV-RNA PCR in necropsy tissues ${ }^{26}$. Only five of these infants were submitted to ZIKV-RNA testing in serum, but no positive test was identified. Thus, congenital ZIKV infection could be laboratory-confirmed in only $7 / 17(41.2 \%)$ infants (Table 2 footnotes).

Adverse pregnancy outcomes according to gestational age of ZIKV maternal infection

Of the 92 women with ZIKV infection in the first trimester, $35.9 \%$ had an adverse pregnancy outcome versus $2.8 \%$ and $1.9 \%$ in the second and third trimesters, respectively (Table 2). Most (76.7\%) of adverse pregnancy outcomes potentially related to ZIKV occurred when the infection was acquired during the first trimester.

Importantly, only one of the seven infants whose mothers had ZIKV infection beyond the first trimester (14 weeks) was classified as typical CZS phenotype. Three of the remaining infants were intrauterine growth restricted what could be related to the moderate microcephaly in whom no brain abnormalities were detected. Two had been congenitally infected with CMV, probably explaining their brain imaging findings, and one had clinical and imaging findings of a possible inborn error of metabolism. None of these infants had positive 
ZIKV results. These 5 infants with other plausible causes for the observed postnatal CNS anomalies were excluded from the typical CZS phenotype classification.

The gestational age of ZIKV infection that best predicted adverse outcomes was 11 completed weeks of pregnancy (Figure 2). The relative risk of adverse pregnancy outcomes potentially related to ZIKV infection was 14.0[95\% CI:7.6-26.0] for maternal infection acquired < 11 weeks as compared to more advanced gestational ages. Gestational age less than eleven completed weeks (11.9) was also the best predictor of the typical CZS phenotype with brain abnormalities. These were much more likely to occur with maternal infection $<11$ weeks of gestation than afterward (relative risk=76.2[95\%CI:10.1-576.6]).

\section{DISCUSSION}

While the ZIKA virus infection outbreak has passed, there is ongoing endemic spread in Brazil, and other countries and women with fetal signs are currently being tested for ZIKV, emphasizing the relevance of broadening available data regarding the vertical transmission of this virus.

\section{Main Findings}

We aimed to investigate adverse pregnancy and early neonatal outcomes according to gestational age of maternal infection in all 511 notified ZIKV-infected women and their offspring from one of the most affected Brazilian regions. Most pregnancies evolved uneventfully. Pregnancy losses occurred in $4.7 \%$ of cases, and abnormalities potentially related to ZIKV infection in $25.0 \%$ of the stillbirths and $3.9 \%$ of the live-born infants. The typical CZS phenotype occurred in $2.9 \%$ of infants (CI95\%:1.7-4.7) without comorbidities. As other congenital infections, the ZIKV congenital disease had a broad spectrum with possible subclinical findings. Apparently asymptomatic infants thoroughly evaluated within 3 months of age had ZIKV-related eye abnormalities (1.3\%), significant imaging brain abnormalities (0.5\%), and potential neurologic/neurodevelopment dysfunctions (8.0\%). Furthermore, early ( $<11$ weeks of gestation) ZIKV maternal infection was strongly associated with the presence of severe neurological impairment.

Strengths and limitations

To the best of our knowledge, this study is the most extensive Brazilian cohort study of women with confirmed ZIKV infection during gestation. There are few large cohorts with robust estimates regarding ZIKV impact on pregnancy losses and fetal malformations. Two early studies of Brazilian cohorts reported very diverse results: -a high prevalence (42\%) of abnormal clinical or imaging findings distributed across trimesters of maternal infection ${ }^{3}$; or -no microcephaly but minor ultrasonography findings aside from ophthalmologic and/or hearing loss in a third of infants ${ }^{8}$. It is possible that referral bias, the relatively small number of mother-infant pairs, and an extensive definition of adverse outcomes might have contributed to the overestimation of the reported effects. Our results were generated in a much more representative sample of the total population once it contained all pregnant women with confirmed symptomatic ZIKV infection living in a distinct geographic region. We used stricter definitions of abnormalities potentially related to ZIKV infection, systematically approached the gestational age of the maternal ZIKV infection as a continuous variable and explored additional etiologies for abnormal infants' findings.

Our cohort set up which allowed obtaining birth data for all of the women with confirmed infection and evaluate most of their infants during the epidemics may be a model to investigate infection-related morbidity in other epidemics such as COVID-19. Further, it demonstrates the need to analyze the gestational age of infection to guide better clinical practice for ZIKV maternal infection and the importance of long-term follow-up for exposed infants, as known for other congenital infections.

Our study does have limitations. Only symptomatic women were selected, and not all infants were followed from birth. Nevertheless, its size is comparable to other country-specific studies, and it contained perinatal outcome data for all women with confirmed ZIKV infection. Infant findings potentially related to ZIKV infection were only considered after ruling out other conditions with a large subgroup of infants having complete clinical, neurological, and brain imaging data. Our results likely reflect the real effect of the maternal ZIKV-symptomatic infection in this population. 


\section{Interpretation}

Reinforcing our findings, four larger cohorts ${ }^{6,7,9,13}$ reported lower estimates of pregnancy losses and/or birth defects $(5-7 \%)$ while we detected $8.4 \%$ of the combined outcomes with an overall agreement among our rate of pregnancy losses $(4.7 \%)$ and these studies (3-10\%). The latter of these cohorts also reported that a laboratory confirmed postnatal diagnosis of CZS, what was not feasible in this study, contributes to $47 \%$ of adverse outcomes and $61 \%$ of severe adverse outcomes described. A metanalysis of studies encompassing 2941 pregnancies $^{27}$ estimated a prevalence of $2.3 \%$ among all pregnancies and $2.7 \%$ in live births, while a recent review showed that among fetuses exposed to ZIKV during pregnancy, fetal losses occurred in 4-7\%, microcephaly in 4-6\% and CZS in $5-14 \%^{2}$, which are similar to this study findings $(4.7 \%, 3.3 \%$ and $2.9 \%$, respectively).

An inverse association between the gestational age of ZIKV infection and the severity of fetal outcomes has been reported with the worst outcomes resulted from first-trimester infection $6,7,9,28-30$. However, no study has yet systematically and prospectively approached the exact gestational age of the ZIKV-infection or explored this as a continuous variable. Most studies estimated maternal infection by trimester ${ }^{3,5-9,29-32}$. Also, only one of the previous studies ${ }^{13}$ has ruled out congenital cytomegalovirus infection based on the detection of CMV-DNA within three weeks of age, which is an important etiology of CNS abnormalities that might confound ZIKV data. We demonstrated that severe adverse outcomes were a consequence of maternal ZIKV infection at $<11$ weeks of gestation. We found no infant with typical CZS whose maternal infection occurred after 14 weeks gestation. Due to the relatively low number of affected infants in this cohort, we could not estimate the influence of potential covariates. Nonetheless, the fact that worst outcomes were 14-fold more likely $<11$ weeks of gestation is a very relevant finding regarding clinical management.

The single study that systematically tested infants born to ZIKV-infected mothers have estimated a 20$30 \%$ maternal-fetal transmission rate ${ }^{13}$. However, versus other congenital infections, confirmation of infant ZIKV infection remains a challenge because no nucleic acid or serological tests have been validated for this purpose. Only a portion of infants with the typical CZS phenotype are positive ${ }^{33}$. Consequently, figures based on detection of nucleic acid or antibodies might underestimate the true vertical transmission rate and all ZIKV-exposed babies should be considered at risk for potential late consequences. We evaluated a subgroup of infants without microcephaly or CNS abnormalities to identify early signs of the disease; $8 \%$ of them had potential neurologic abnormalities. Indeed, CNS structural findings related to ZIKV infection can be silent in the neonatal period for infants not submitted to neuroimaging examinations ${ }^{34}$. However, less is known about isolated early warning signs of neurological dysfunctions in young infants without microcephaly and CNS malformations. Prior work showed that $18 \%^{35}$ to $50 \%^{36}$ infants had neurological findings. However, some of these infants had obvious CNS abnormalities limiting comparisons with our study. We continue to follow the infants to confirm the persistence or appearance of neurodevelopmental findings as suggested by others ${ }^{12,37}$. Certainly, upcoming controlled cohort studies are needed for a better understanding of the long-term adverse outcomes of infants born to ZIKV-infected pregnant women.

\section{CONCLUSION}

Our study demonstrated that short-term adverse outcomes potentially related to ZIKV in infants born to ZIVK-infected symptomatic women are less frequent (2.5-6\%) than previously thought. A strong relationship between the $<11$ weeks gestational age of maternal infection and worse prognosis was demonstrated. Up to $10 \%$ of apparently asymptomatic infants during the first three months of life can exhibit neurological, cranial ultrasonography and or ophthalmologic abnormalities. This stresses the importance of using preventive measures in the early phases of pregnancy and set the basis for unstrained advice for pregnant women who acquire ZIKV infection after 11 weeks of gestation. Until results from controlled studies on the definition of medium and long-term prognosis for ZIKV-exposed fetuses are available, a thorough postnatal follow-up for these newborns is strongly recommended.

Acknowledgments : The authors are grateful to all members of the Epidemiology and Disease Control Division of Ribeirão Preto's Secretary of Health, especially Luzia Márcia Romanholi Passos, for their important 
contribution regarding prenatal data of ZIKV symptomatic pregnant patients. We acknowledge the support of the Pediatric Infectious Diseases Laboratory technicians and the staff members of the Study Center on Maternal, Perinatal and Infant's Infection from Ribeirão Preto Medical School (NEIMPI).

Disclosure of interests: All manuscript authors (C.M.C., S.F.B.M.N., D.C.A.A., S.R.T., F.R.A., M.C.R.M., J.D.C.P.F., M.S.F.M., B.V.M.N., C.A.C.T.C., A.R.T.A., J.M.F., A.A.T.B., A.Y.Y., G.D., M.M.M.P.) confirm that they don't have any conflicts of interest to declare.

Contribution to authorship: C.M.C.: Conceptualization, Methodology, Formal analysis, Investigation, Writing - Original Draft, Writing - Review \& Editing. S.F.B.M.N.: Formal analysis, Investigation, Writing - Review \& Editing. D.C.A.A.: Formal analysis, Investigation, Writing - Review \& Editing. S.R.T.: Formal analysis, Investigation, Writing - Review \& Editing. F.R.A.: Investigation, Writing - Review \& Editing. M.C.R.M.: Formal analysis, Investigation, Writing - Review \& Editing. J.D.C.P.F.: Investigation, Writing Review \& Editing. M.S.F.M.: Investigation, Writing - Review \& Editing. B.V.M.N.: Investigation, Writing - Review \& Editing. C.A.C.T.C.: Formal analysis, Investigation, Writing - Review \& Editing. A.R.T.A.: Formal analysis, Investigation, Writing - Review \& Editing. J.M.F.: Formal analysis, Investigation, Writing - Review \& Editing. A.A.T.B.: Project administration, Writing - Review \& Editing. A.Y.Y.: Conceptualization, Methodology, Formal analysis, Writing - Review \& Editing. G..: Conceptualization, Methodology, Writing - Original Draft, Writing - Review \& Editing, Supervision. M.M.M-P.: Conceptualization, Methodology, Formal analysis, Writing - Original Draft, Writing - Review \& Editing, Supervision, Project administration, Funding acquisition.

\section{Appendix: The NATZIG Cohort Study members}

Obstetrics : Geraldo Duarte, Conrado Milani Coutinho, Patrícia Pereira dos Santos Melli, Marília Carolina Razera Moro, Ligia Conceição Marçal Assef, Greici Schroeder, Silvana Maria Quintana.Pediatrics : Marisa Márcia Mussi-Pinhata, Adriana Aparecida Tiraboschi Bárbaro, Juliana Dias Crivelenti Pereira Fernandes, Márcia Soares Freitas da Motta, Fabiana Rezende Amaral, Paulo Henrique Manso, Bento Vidal de Moura Negrini, Daniela Anderson, Juannicelle Tenório Albuquerque Madruga Godoi, Marina de Mattos Louren. Pathology : Fernando da Silva Ramalho. Neuropediatrics: Ana Paula Andrade Hamad, Carla Andréa Cardoso Tanuri Caldas, Marili André Coelho, Rafaela Pichini de Oliveira. Neurodevelopment : Silvia Fabiana Biason de Moura Negrini, Stephani Ferreira Rodrigues, Nádia Lombardi Maximino Siqueira, Danusa Menegat, Thamires Máximo Neves Felice.Ophthalmology : João Marcello Fortes Furtado, Milena Simões Freitas e Silva, Rafael Estevão De Angelis. Audiology : Adriana Ribeiro Tavares Anastasio, Evelin Fernanda Teixeira, Cristiane Silveira Guidi, Priscila Morales Andreazzi. Neuroimaging : Sara Reis Teixeira, Jorge Elias Junior, Antônio Carlos dos Santos. Social Service : Adriana Veneziani Morales Morimoto, Joseane Cristina Bonfim Augusto. Research Nurses : Maria Natalina Ferreira da Silva, Eunice Gonçalves da Silva, Maria Beatriz Cruz de Souza.Laboratory support : Aparecida Yulie Yamamoto, Cleonice de Souza Barbosa Sandoval, Mirian Borges de Oliveira, Alessandra Santos Zampolo.

\section{Details of Ethics Approval}

All study procedures received ethical approval on the $24^{\text {th }}$ of June 2016 (Processes \#7404/2016, \#5914/2017) by Clinics Hospital, Ribeirão Preto School of Medicine, Ribeirão Preto, SP, Brazil, Institutional Ethics Committee. Written informed consent was obtained from all participants.

\section{Funding}

This study was mainly supported by FAEPA, Brazil, and received partial support from the CVE SES-SP. The funding sources didn't have any role in the writing, the decision to submit the manuscript for publication or any other aspect pertinent to study conduction. There was no funding from pharmaceutical companies or other agencies. All study data were analyzed by the corresponding author, as well as the final responsibility for the decision to submit for publication.

\section{REFERENCES}


1. Pomar L, Musso D, Malinger G, Vouga M, Panchaud A, Baud D. Zika virus during pregnancy: From maternal exposure to congenital Zika virus syndrome. Prenat Diagn. 2019;39(6):420-430.

2. Musso D, Ko AI, Baud D. Zika Virus Infection - After the Pandemic.N Engl J Med. 2019;381(15):14441457 .

3. Brasil P, Pereira JP, Jr., Moreira ME, et al. Zika Virus Infection in Pregnant Women in Rio de Janeiro. $N$ Engl J Med.2016;375(24):2321-2334.

4. Ellington SR, Devine O, Bertolli J, et al. Estimating the Number of Pregnant Women Infected With Zika Virus and Expected Infants With Microcephaly Following the Zika Virus Outbreak in Puerto Rico, 2016. JAMA Pediatr. 2016;170(10):940-945.

5. Reynolds MR, Jones AM, Petersen EE, et al. Vital Signs: Update on Zika Virus-Associated Birth Defects and Evaluation of All U.S. Infants with Congenital Zika Virus Exposure - U.S. Zika Pregnancy Registry, 2016. MMWR Morb Mortal Wkly Rep. 2017;66(13):366-373.

6. Honein MA, Dawson AL, Petersen EE, et al. Birth Defects Among Fetuses and Infants of US Women With Evidence of Possible Zika Virus Infection During Pregnancy. JAMA. 2017;317(1):59-68.

7. Shapiro-Mendoza CK, Rice ME, Galang RR, et al. Pregnancy Outcomes After Maternal Zika Virus Infection During Pregnancy - U.S. Territories, January 1, 2016-April 25, 2017. MMWR Morb Mortal Wkly Rep.2017;66(23):615-621.

8. Nogueira ML, Nery Junior NRR, Estofolete CF, et al. Adverse birth outcomes associated with Zika virus exposure during pregnancy in Sao Jose do Rio Preto, Brazil. Clin Microbiol Infect.2018;24(6):646-652.

9. Hoen B, Schaub B, Funk AL, et al. Pregnancy Outcomes after ZIKV Infection in French Territories in the Americas. N Engl J Med.2018;378(11):985-994.

10. Conners EE, Lee EH, Thompson CN, et al. Zika Virus Infection Among Pregnant Women and Their Neonates in New York City, January 2016-June 2017. Obstet Gynecol. 2018;132(2):487-495.

11. Adamski A, Bertolli J, Castaneda-Orjuela C, et al. Estimating the numbers of pregnant women infected with Zika virus and infants with congenital microcephaly in Colombia, 2015-2017. J Infect.2018;76(6):529535.

12. Rice ME, Galang RR, Roth NM, et al. Vital Signs: Zika-Associated Birth Defects and Neurodevelopmental Abnormalities Possibly Associated with Congenital Zika Virus Infection - U.S. Territories and Freely Associated States, 2018. MMWR Morb Mortal Wkly Rep.2018;67(31):858-867.

13. Pomar L, Vouga M, Lambert V, et al. Maternal-fetal transmission and adverse perinatal outcomes in pregnant women infected with Zika virus: prospective cohort study in French Guiana. BMJ. 2018;363:k4431.

14. WHO. Zika Epidemiology Update, July 2019. In. https://www.who.int/docs/defaultsource/documents/emergencies/zika/zika-epidemiology-update-july-2019.pdf?sfvrsn=14a1b3a7_22019.

15. El-Sayed A, Kamel M. Climatic changes and their role in emergence and re-emergence of diseases. Environ Sci Pollut Res Int . 2020.

16. Instituto Brasileiro de Geografia e Estatística (IBGE) (28th August 2019). «Estimativas da população residente nos municípios brasileiros com data referência em $1^{0}$ de julho de 2019» (PDF). Accessed on 28th August 2019.

17. Lanciotti RS, Kosoy OL, Laven JJ, et al. Genetic and serologic properties of Zika virus associated with an epidemic, Yap State, Micronesia, 2007. Emerg Infect Dis. 2008;14(8):1232-1239.

18. Committee on Practice B-O, the American Institute of Ultrasound in M. Practice Bulletin No. 175: Ultrasound in Pregnancy. Obstet Gynecol. 2016;128(6):e241-e256. 
19. Villar J, Cheikh Ismail L, Victora CG, et al. International standards for newborn weight, length, and head circumference by gestational age and sex: the Newborn Cross-Sectional Study of the INTERGROWTH-21st Project. Lancet. 2014;384(9946):857-868.

20. Papageorghiou AT, Ohuma EO, Altman DG, et al. International standards for fetal growth based on serial ultrasound measurements: the Fetal Growth Longitudinal Study of the INTERGROWTH-21st Project.Lancet. 2014;384(9946):869-879.

21. EUROCAT Guide 1.4: Instruction for the registration of congenital anomalies. EUROCAT Central Registry, University of Ulster; 2013. https://eu-rdplatform.jrc.ec.europa.eu/sites/default/files/Full\%20Guide\%201\%204\%20version\%2003_OCT2018.pdf. Accessed 03/08/2019.

22. Dubowitz L, Mercuri E, Dubowitz V. An optimality score for the neurologic examination of the term newborn. J Pediatr.1998;133(3):406-416.

23. Bayley N. Bayley Scales of Infant and Toddler Development -Third Edition: Screening Test Manual. In. San Antonio, Texas: Pearson Clinical Assessment PsychCorp; 2006:146.

24. Mercuri E, Ricci D, Pane M, Baranello G. The neurological examination of the newborn baby. Early Hum Dev.2005;81(12):947-956.

25. Speybroeck N. Classification and regression trees. Int J Public Health. 2012;57(1):243-246.

26. Ramalho FS, Yamamoto AY, da Silva LL, et al. Congenital Zika Virus Infection Induces Severe Spinal Cord Injury. Clin Infect Dis.2017;65(4):687-690.

27. Coelho AVC, Crovella S. Microcephaly Prevalence in Infants Born to Zika Virus-Infected Women: A Systematic Review and Meta-Analysis.Int J Mol Sci. 2017;18(8).

28. Pacheco O, Beltran M, Nelson CA, et al. Zika Virus Disease in Colombia - Preliminary Report. $N$ Engl J Med. 2016.

29. Lima GP, Rozenbaum D, Pimentel C, et al. Factors associated with the development of Congenital Zika Syndrome: a case-control study. BMC Infect Dis. 2019;19(1):277.

30. Brady OJ, Osgood-Zimmerman A, Kassebaum NJ, et al. The association between Zika virus infection and microcephaly in Brazil 2015-2017: An observational analysis of over 4 million births. PLoS Med.2019;16(3):e1002755.

31. Rodriguez-Morales AJ, Cardona-Ospina JA, Ramirez-Jaramillo V, et al. Diagnosis and outcomes of pregnant women with Zika virus infection in two municipalities of Risaralda, Colombia: Second report of the ZIKERNCOL study. Travel Med Infect Dis. 2018;25:20-25.

32. Subissi L, Dub T, Besnard M, et al. Zika Virus Infection during Pregnancy and Effects on Early Childhood Development, French Polynesia, 2013-2016. Emerg Infect Dis. 2018;24(10):1850-1858.

33. de Araujo TVB, Rodrigues LC, de Alencar Ximenes RA, et al. Association between Zika virus infection and microcephaly in Brazil, January to May, 2016: preliminary report of a case-control study.Lancet Infect Dis. 2016;16(12):1356-1363.

34. Aragao M, Holanda AC, Brainer-Lima AM, et al. Nonmicrocephalic Infants with Congenital Zika Syndrome Suspected Only after Neuroimaging Evaluation Compared with Those with Microcephaly at Birth and Postnatally: How Large Is the Zika Virus "Iceberg"? AJNR Am J Neuroradiol. 2017;38(7):1427-1434.

35. Einspieler C, Utsch F, Brasil P, et al. Association of Infants Exposed to Prenatal Zika Virus Infection With Their Clinical, Neurologic, and Developmental Status Evaluated via the General Movement Assessment Tool. JAMA Netw Open. 2019;2(1):e187235. 
36. Cardoso TF, Jr., Santos RSD, Correa RM, et al. Congenital Zika infection: neurology can occur without microcephaly. Arch Dis Child. 2019;104(2):199-200.

37. Lopes Moreira ME, Nielsen-Saines K, Brasil P, et al. Neurodevelopment in InfantsExposed to Zika Virus In Utero. $N$ Engl $J$ Med. 2018;379(24):2377-2379.

\section{TABLE/FIGURE CAPTION LIST}

Figure 1 - Flowchart showing ZIKV-exposed neonates and infants enrolled in the study and perinatal results (CZS malformations: malformations potentially related to congenital Zika virus infection. HCRP: Referral hospital).

Figure 2 - Conditional inference tree associating gestational age to adverse pregnancy outcomes. Study subjects were classified according to the presence of any adverse outcomes potentially related to ZIKV infection (miscarriages, stillbirths, microcephaly with or without brain abnormalities and brain abnormalities without microcephaly).

Table 1 - Major abnormalities diagnosed among 489 live-born neonates.

Table 2 - Adverse pregnancy and neonatal outcomes potentially related to ZIKV infection according to trimester of maternal infection.

Table S1 - General and ZIKV infection-related characteristics of 511 pregnant women.

Table S2 - Pregnancy outcomes of 511 women with confirmed ZIKV infection and general characteristics of their 489 live born infants.

Table S3 - Comparison of maternal and infants' characteristics of asymptomatic newborns additionally assessed within three months of life with those of not assessed newborns.

\section{Hosted file}

Tables.docx available at https://authorea.com/users/328445/articles/455724-earlymaternal-zika-infection-predicts-severe-neonatal-neurological-damage-results-from-theprospective-natural-history-of-zika-virus-infection-in-gestation-natzig-cohort-study 

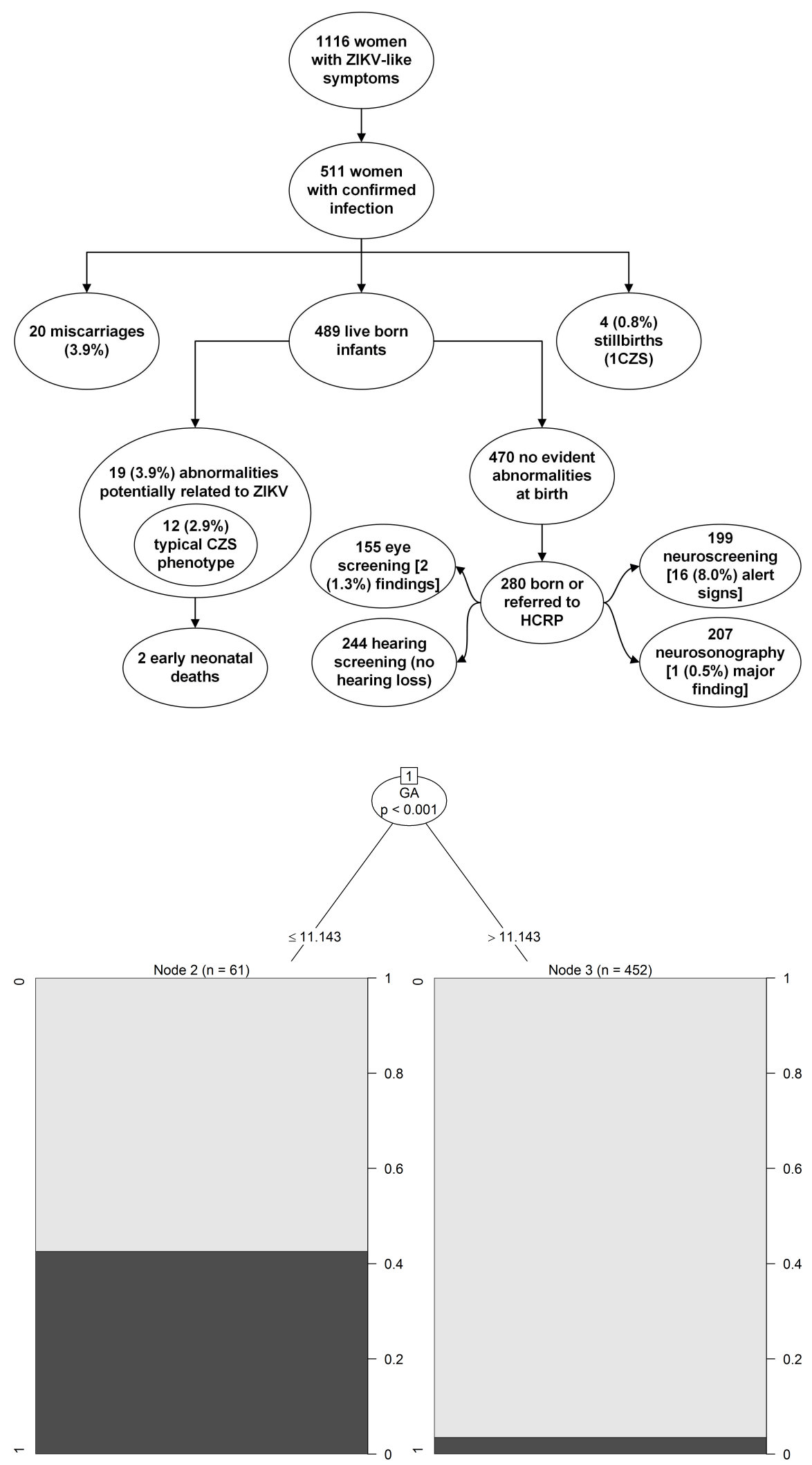